\title{
Awareness of the Complications from Impacted Third Molar Surgeries among General Dental Practitioners
}

\author{
Farokh Farhadi ${ }^{1}$, Ahad Navidi2* ${ }^{*}$ Ramin Maheri ${ }^{3}$ \\ 1. Department of Oral and Maxillofacial Surgery, Faculty of Dentistry, Tabriz University of Medical Sciences, Tabriz, \\ Iran \\ 2. Faculty of Dentistry, Tabriz University of Medical Sciences, Tabriz, Iran \\ 3. Dental Branch of Tehran Azad University, Tehran, Iran
}

\begin{abstract}
Introduction: Surgery of impacted third molars and the resultant complications are common occurrences in dental offices. Therefore, the present study was undertaken to determine the awareness of general dental practitioners in Tabriz of complications of surgeries of impacted third molars. Materials and methods: In the present study a researcher-made questionnaire was completed by 186 randomly selected general dentists in Tabriz. After collecting the questionnaires and extractions of data, descriptive statistical methods and chi-squared test were used to evaluate the relationship between personal demographic variables (independent) and the dependent variable of the study with SPSS 14 . Statistical significance was set at $P<0.05$. Results: The mean \pm standard deviation of the awareness of the subjects of complications of surgery of impacted third molars was $9.87 \pm 2.66$, with minimum and maximum scores of 2 and 16, respectively. Of 186 dentists included in the study, 15 (8.1\%) had low awareness, 152 (81.7\%) had moderate awareness and 19 (10.2\%) had high awareness. Chi-squared test showed no significant relationship between awareness and other variables (age, gender and job experience) ( $\mathrm{P}>0.05)$. Conclusion: Based on the results, the awareness of general dental practitioners in Tabriz of the complications of impacted third molar surgeries was at a moderate level.
\end{abstract}

Key words: Awareness; general dental practitioner; impacted third molar; complications;

\section{Introduction}

Extraction of third molars is a common procedure in the field of oral and maxillofacial surgery. The reasons for extraction of these teeth include acute or chronic pericoronitis, presence of a cyst or tumor, periodontal problems and carious lesions in the second or third mandibular molars (1). In some cases, these teeth are extracted for orthodontic treatment or orthognathic surgeries. In some other cases these extractions are carried out to avoid problems in future. Before any action to extract these teeth, the patients should be informed of the procedure.

\section{Corresponding author:}

Ramin Maheri

Dental Branch of Tehran Azad University, Tehran, Iran

Email: dr_rama_75@yahoo.com Phone: +989143136141

Receive date: 2016-02-3| Accept date: 2016-03-27| Publish date: 2016-04-19

DOI: 10.7575/aiac.abcmed.16.04.02.05 
Extraction of impacted mandibular third molars is associated with some problems the most prevalent of which are alveolitis, infection and paresthesia of the inferior alveolar nerve $(2,4)$. Hemorrhage during or after surgery and paresthesia of the lingual nerve are rather rare $(3,5)$. It appears the surgical technique has an important role in the last complication mentioned above (6). Most these problems are temporary but in some cases paresthesia might be permanent and result in more problems (68).

Many factors are associated with these problems, including age, patient health, impaction severity, the surgeon's experience, the surgical technique used, smoking and use of oral contraceptives $(4,7)$. Based on previous studies, the first three factors mentioned above have a strong relationship with the complications (3-5).

Undoubtedly, awareness and knowledge of dentists are the most important factors in managing dental emergencies and events during and after treatment and in determining the actions taken. Several studies have been carried out in different parts of the world in relation to determining the awareness of general dental practitioners about complications of surgeries in the oral cavity. These studies on the awareness of dentists in relation to managing dental emergencies have been related to prophylactic antibiotic therapy and infective endocarditis, complications of local anesthesia, and managing and drug therapy (antimicrobial treatment) of third molars. The results of these studies have shown insufficient knowledge of dentists about these complications in most cases (9-12).

Considering the relatively high rate of complications of impacted third molar surgeries and since the awareness of general dental practitioners of the related issue has not been satisfactory and no studies have been carried out to date to determine the awareness of dentist about these issues, the present study was carried out to evaluate the awareness of general dental practitioners in Tabriz of the problems associated with surgeries of impacted third molars.

\section{Materials and Methods}

The study community consisted of general dental practitioners with private offices in Tabriz. A total of 350 general dental practitioners have offices in Tabriz based on statistics provided by the Medical Organization, Tabriz Branch. The list of the dentists was prepared and simple random sampling technique was used to select 186 dentists. Cochrane sampling formula was used to determine the sample size.

First a questionnaire was designed. The questions were in the open and closed formats. The questions were divided into two sections. The first section consisted of questions on demographic data and personal information, including age, gender, job experience, and the second section consisted of questions on dentist's awareness of the complications of surgical extraction of impacted third molars. To evaluate the content validity of the questionnaire, it was submitted to three academic staff members of the Faculty and any questions requiring modifications were modified. After the questionnaire was designed it was handed in to 20 dentists in a pilot study. The questionnaire was submitted to the dentists in an envelope by directly referring to the dental office. The dentists were reassured that the data on the questionnaire would be kept confidential and it was not necessary to write any names on the questionnaires. The next day the questionnaires were collected and the questions were evaluated. Then inappropriate questions were removed the revised questionnaire was primed in adequate numbers. Each correct response received a score of 1 and incorrect responses did not receive any scores. Then the total score of each 
dentist was determined and reported as the awareness score.

After 20 questionnaires were completed the dentists, the reliability of the questions was determined based on Cronbach's alpha. Data were analyzed with SPSS 14 using descriptive statistics and the results were reported as frequency percentages and means \pm SD. Finally, chi-squared test and Fisher's exact test were used to evaluate the relationship between demographic variables (independent) and the study's dependent variable. Statistical significance was defined at $\mathrm{P}<0.05$.

\section{Results}

First the reliability of the questions was evaluated. The reliability of the questions was over 0.6 , indicating the scientific validity of variables.

The mean \pm SD of the dentist's awareness scores in relation to complications of impacted third molar surgeries was $9.87 \pm 2.66$, with minimum and maximum scores of 2 and 16, respectively. In this context, $25 \%$ of the subjects had scores under $8,25 \%$ had scores $8-$ $10,25 \%$ had scores $10-12$ and $25 \%$ had scores over 12. On the whole, the awareness of dentists of complications of impacted third molar surgeries was at a moderate level (Graph 1). Of 186 dentists included in the study, 15 (8.1\%) had low awareness, 152 (81.7\%) had moderate awareness and 19 (10.2\%) had a high level of awareness (Graph 2).

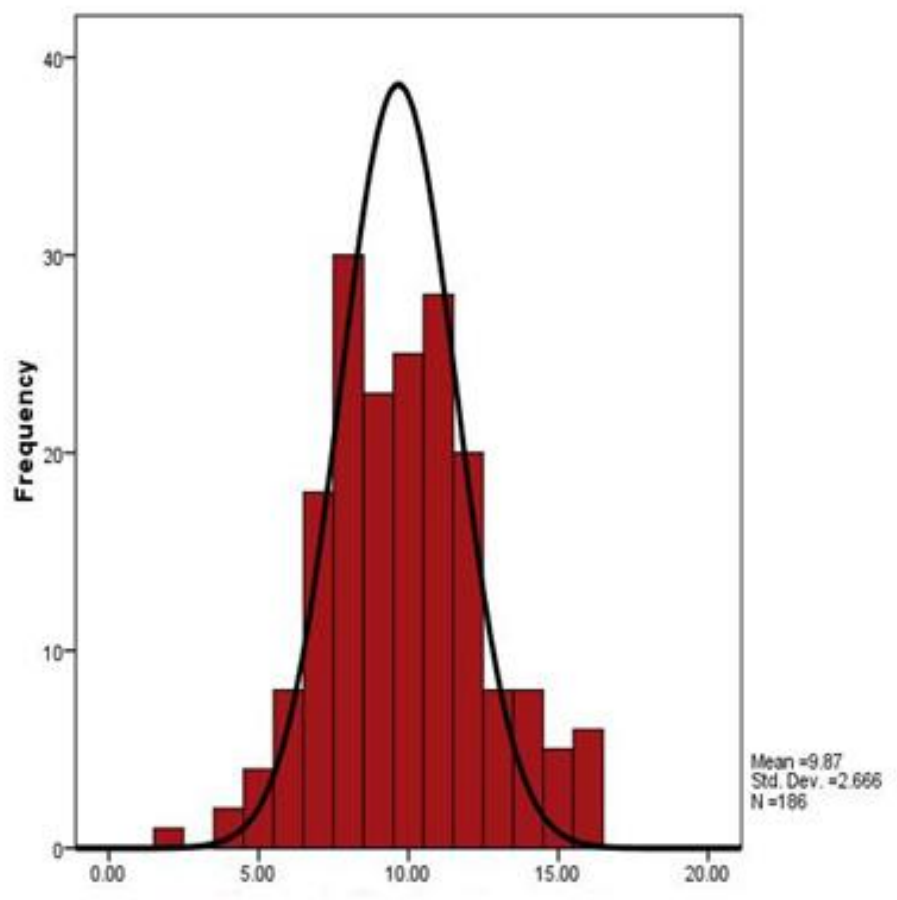

Graph 1: Dispersion the awareness of the dentists evaluated in the present study of the complications of impacted third molar surgeries 


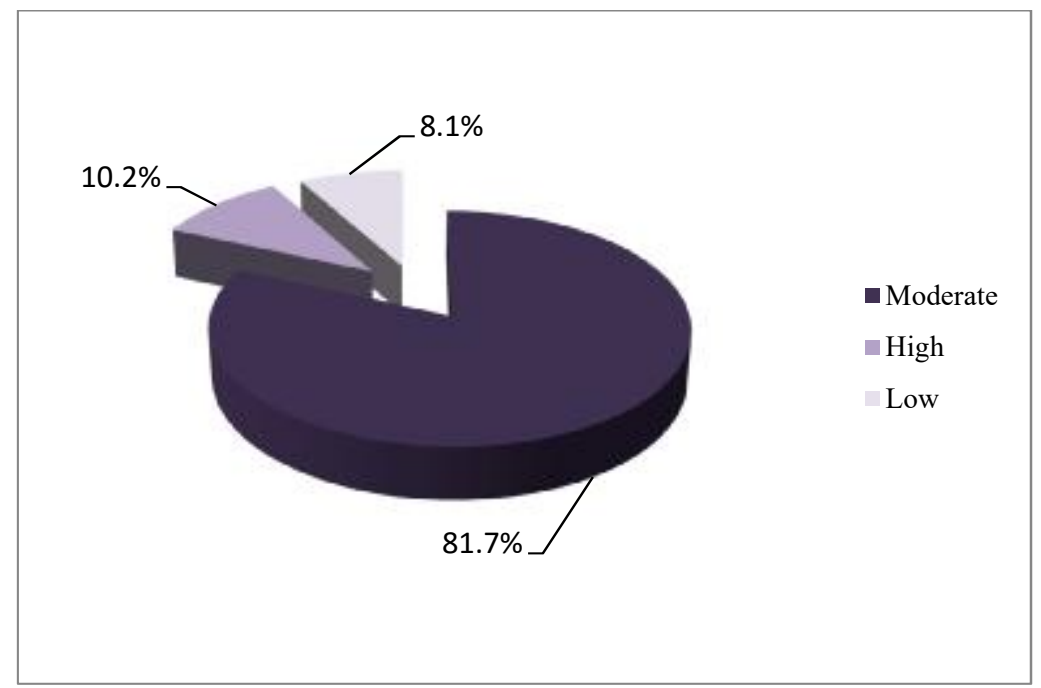

Graph 2: Frequency distribution of the dentists' awareness evaluated in the present study of the complications of impacted third molar surgeries

Evaluation of the awareness of dentists in terms of gender by chi-squared test showed no significant differences in the awareness of surgeries between males and females $(P>0.05$, $\chi 2=0.920)$ (Graph 3). dentists of complications of third molar

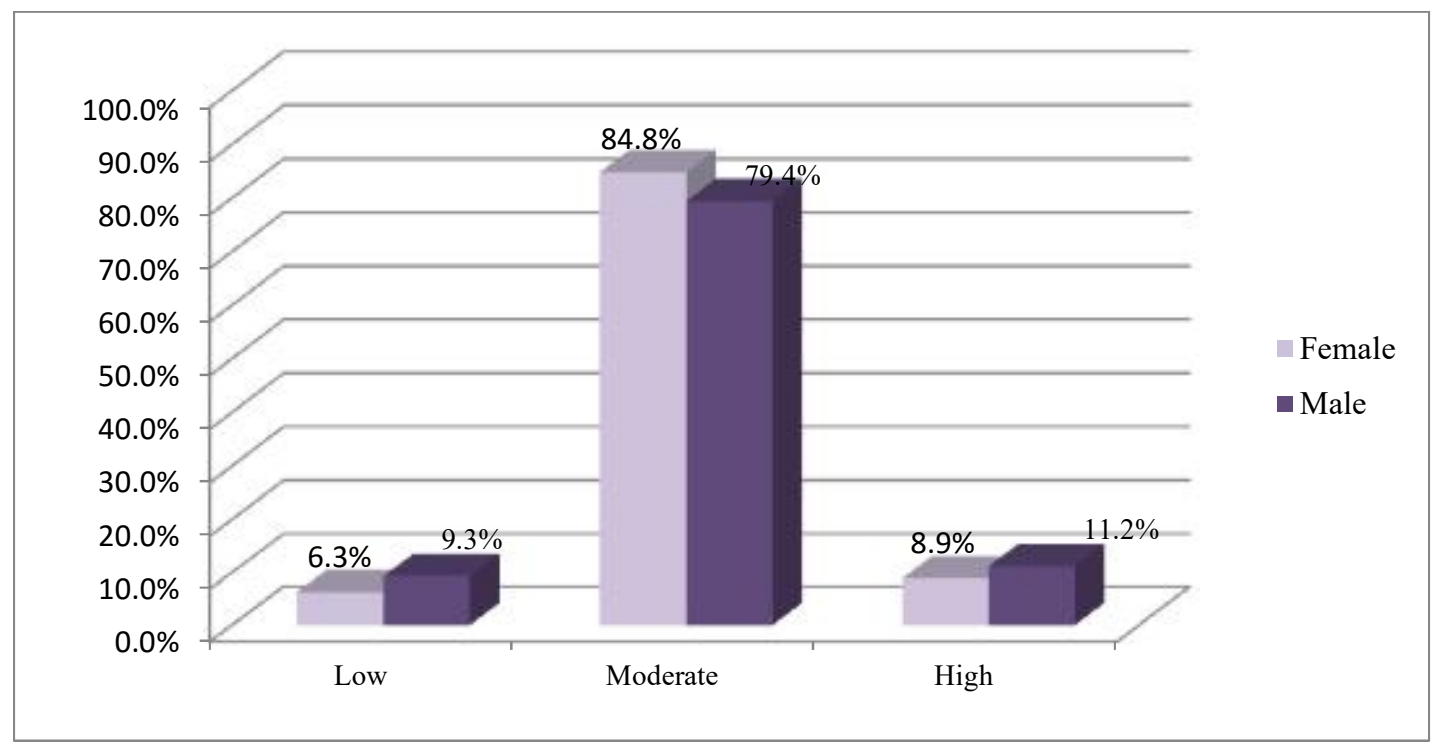

Graph 3: Comparison of the awareness of dentists in terms of gender.

Evaluation of the awareness of dentists in terms of age by chi-squared test showed no significant differences in the awareness of 
dentists of complications of third molar $(P>0.05, \chi 2=2.94)($ Graph 4). surgeries between different age groups

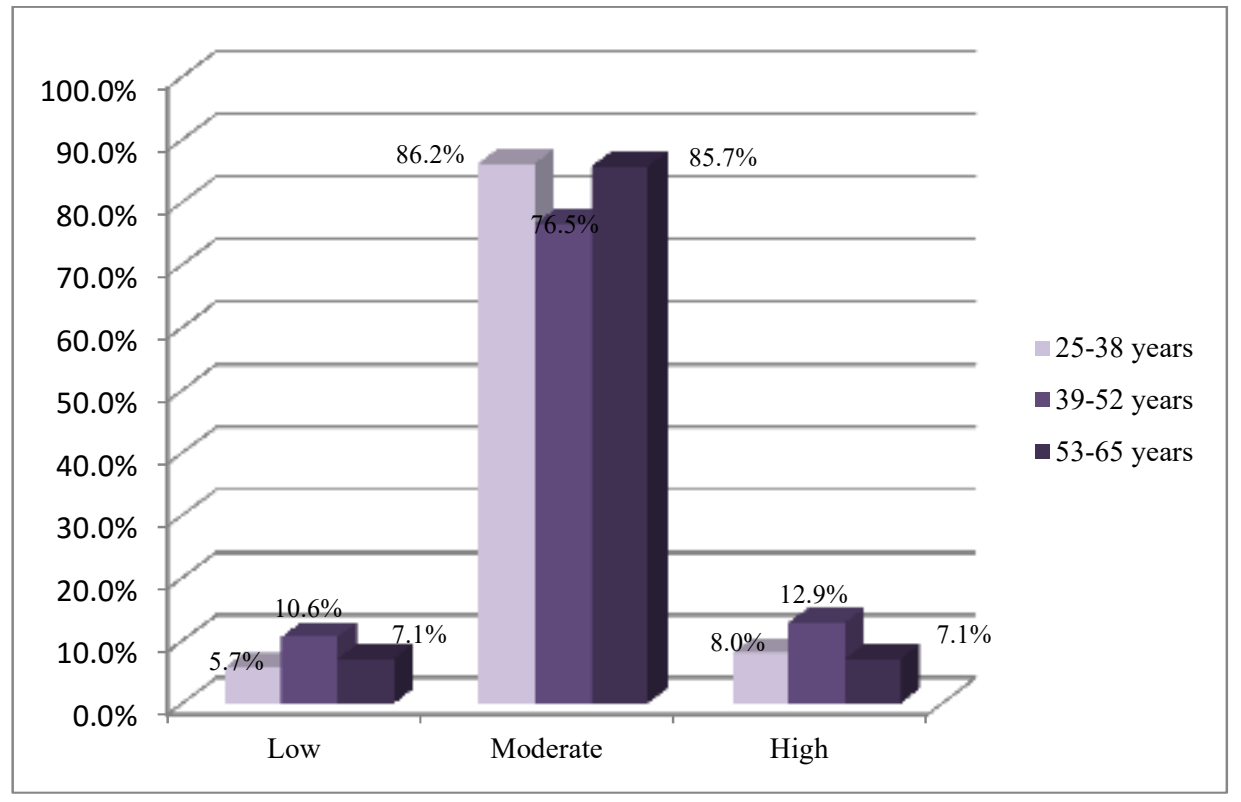

Graph 4: Comparison of the awareness of dentists in terms of age

third molar surgeries in terms of job experience

Evaluation of the awareness of dentists in $(P>0.05, \chi 2=4.42)($ Graph 5). terms of job experience by chi-squared test showed no significant differences in the awareness of dentists about complications of

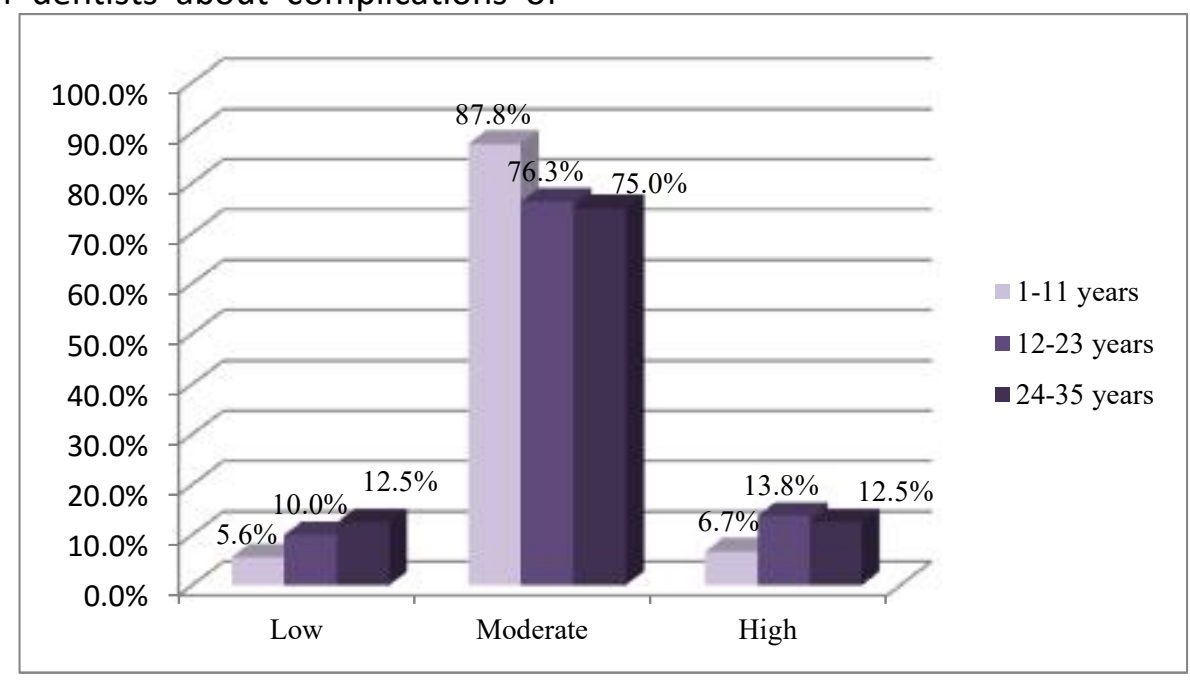

Graph 5: Comparison of the awareness of dentists in terms of job experience 


\section{Discussion}

The awareness of general dental practitioners in Tabriz of the complications of impacted third molar surgeries was evaluated in the present study. The present study was undertaken considering the relatively high prevalence of complications after impacted third molar surgeries and regarding the unfavorable level of awareness of general dental practitioners of the relevant issues and since no study to data has evaluated the awareness of dentists in this field.

The results collected from the questionnaires completed by the dentists included in the present study showed a moderate level of awareness of complications after impacted third molar surgeries. The results showed a high level of awareness in relation to the most common complications, the duration of the problems, the possibility of the tooth being pushed into anatomic spaces, the techniques used to extract the tooth and complications associated with not removing a short and sound root which has been fractured. Awareness in relation to formation of oroantral communication and the technique used to close it and also the various methods and appropriate materials in different forms of hemorrhage was at a low to moderate level. Deductive findings showed no significant relationship between awareness and other variables, including age, gender and jobs experience.

Undoubtedly, dentists' knowledge is very important for managing dental emergencies intra-operatively and post-operatively and can determine their performance during such incidents. No studies have evaluated dentists' awareness of complications of surgeries of impacted third molars. However, several studies have been carried out all over the world to determine dentists' awareness of complications of surgeries in the oral cavity. These studies have shown low levels of awareness in relation to management of medical emergencies, prophylactic antibiotic therapy and infective endodontists, complications of local anesthesia, and management and antimicrobial drug therapy of third molars. The results of these studies have shown inadequate awareness in these fields in the majority of cases (9-12).

Centinkaya et al evaluated of dentists' awareness of anaphylaxis due to local anesthesia and reported inadequate awareness of dentists' of allergies and anaphylaxis in relation to the use of local anesthetic agents, suggesting that it was necessary to design continuous education programs in this context (9).

Zadik et al evaluated dentists' awareness of the guidelines of American Heart Association in relation to prevention of infective endocarditis. The results showed a high level of awareness of the guidelines and their acceptance of the guidelines (10).

\section{Conclusion}

Based on the results of the present descriptive study, awareness of general dental partitions in Tabriz of the complications of surgeries of impacted third molars was at a moderate level. No significant relationship was found between awareness and variables such as age, gender and job experience.

\section{References}

1. Chiapasco M, De Cicco L, Marrone G (1993) Side effects and complications associated with third molar surgery. Oral Surg Oral Med Oral Pathol 76:412-420. 
2. Mercier P, Precious D (1992) Risks and benefits of removal of impacted third molars. A critical review of the literature. Int J Oral Maxillofac Surg 21:17-27.

3. Sisk AL, Hammer WB, Shelton DW, Joy ED, Jr. (1986) Complications following removal of impacted third molars: the role of the experience of the surgeon. J Oral Maxillofac Surg 44:855-859.

4. Bui CH, Seldin EB, Dodson TB (2003) Types, frequencies, and risk factors for complications after third molar extraction. J Oral Maxillofac Surg 61:1379-1389.

5. Muhonen A, Venta I, Ylipaavalniemi P (1997) Factors predisposing to postoperative complications related to wisdom tooth surgery among university students. J Am Coll Health 46:39-42.

6. Brann CR, Brickley MR, Shepherd JP (1999) Factors influencing nerve damage during lower third molar surgery. $\mathrm{Br}$ Dent J 186:514-516.

7. Lopes V, Mumenya R, Feinmann C, Harris M (1995) Third molar surgery: an audit of the indications for surgery, postoperative complaints and patient satisfaction. Br J Oral Maxillofac Surg 33:33-35.

8. Nickel AA, Jr. (1990) A retrospective study of paresthesia of the dental alveolar nerves. Anesth Prog 37:42-45.

9. Cetinkaya F, Sezgin G, Aslan OM (2010) Dentists' knowledge about anaphylaxis caused by local anaesthetics. Allergol Immunopathol (Madr).

10. Zadik Y, Findler M, Livne S, Levin L, Elad S (2008) Dentists' knowledge and implementation of the 2007 American Heart Association guidelines for prevention of infective endocarditis. Oral Surg Oral Med Oral Pathol Oral Radiol Endod 106:e16-19.

11. Adewole RA, Sote EO, Oke DA, Agbelusi AG (2009) An assessment of the competence and experience of dentists with the management of medical emergencies in a Nigerian teaching hospital. Nig Q J Hosp Med 19:190-194.

12. Wilhelm RJ, Sutley SH, Quigley NC (1996) Antimicrobial management of third molars: survey results for military dentists. Gen Dent 44:538-543.

13. Khanuja A, Power MP (2005) Surgical management of impacted teeth. In: Fonseca RJ (ed) Oral and maxillofacial surgery. Saunders, pp 245-280.

14. Meurman JH, Rajasuo A, Murtomaa H, Savolainen S (1995) Respiratory tract infections and concomitant pericoronitis of the wisdom teeth. BMJ 310:834-836.

15. Peterson LJ (2008) Surgical management of impacted teeth. In: Peterson LJ, Indresano AT, Marciani RD, Roser SM (eds) Principles of oral and maxillofacial surgery. Lippincott, pp 103-122.

16. Brickley M, Kay E, Shepherd JP, Armstrong RA (1995) Decision analysis for lower-third-molar surgery. Med Decis Making 15:143-151.

17. Glosser JW, Campbell JH (1999) Pathologic change in soft tissues associated with radiographically 'normal' third molar impactions. Br J Oral Maxillofac Surg 37:259-260.

18. Peterson LJ (2007) Priciples of management of impacted teeth. In: Peterson LJ, Ellis E, Hupp JR, Tucker MR (eds) Contemporary oral and maxillofacial surgery. Mosby, St. Louise, pp 215-247

19. Malamed SF (2004) Handbook of local anesthesia. Mosby, St. Lousie, p 200. 